\title{
SUBDIFFERENTIALS ARE LOCALLY MAXIMAL MONOTONE
}

\section{S. Simons}

In a recent paper, Fitzpatrick and Phelps introduced a new class of operators on a Banach space, the locally maximal monotone operators, and showed that this kind of operator can be approximated by a sequence of nicer maximal monotone operators. We give here an affirmative answer to a question posed in this paper: is the subdifferential of a proper convex lower semicontinuous function necessarily locally maximal monotone? Since a locally maximal operator is maximal monotone, our result represents a strengthening of Rockafellar's maximal monotonicity theorem.

In a recent paper, Fitzpatrick and Phelps [2] introduced a new class of operators on a Banach space, the locally maximal monotone operators, and showed that this kind of operator can be approximated by a sequence of nicer maximal monotone operators. It is clear from its definition that a locally maximal monotone operator is maximal monotone. On the other hand, Fitzpatrick and Phelps pointed out in [2, Theorem 3.5] that the range of a locally maximal monotone operator has a convex closure, and hence that an example of Gossez (see [3]), shows that a maximal monotone operator is not necessarily locally maximal monotone. [2] left open the question whether the subdifferential of a proper convex lower semicontinuous function is necessarily locally maximal monotone. In this note, we shall give an affirmative answer to this question. It follows from the above comments that our result represents a strengthening of Rockafellar's maximal monotonicity theorem.

Lemmas 2-4 will be proved in greater generality that is actually required for the Main Theorem. Specifically, they will be phrased in terms of a sublinear functional $T$ satisfying only (0.1). (This greater generality does not seem to require any extra work; on the contrary, it probably allows us to simplify the notion somewhat). For the Main Theorem, we shall apply these Lemmas in the case where $T$ is given by a simple specific formula (see Lemma 5).

A word is in order about two proofs that we have given recently of the maximal monotonicity theorem. The first (see [4]) relies on the formula for the subdifferential of a sum, while the second (see [5]) relies on manipulating sublinear functionals or

\section{Received 11 June 1992}

The author would like to thank Professor R.R. Phelps for s number of helpful suggestions about earlier drafts of this paper.

Copyright Clearance Centre, Inc. Serial-fee code: 0004-9728/93 \$A2.00+0.00. 
extended sublinear functionals (that is to say, sublinear functionals that can possibly take the value $+\infty$ ). The advantage of the second method is that it is a more or less mechanical procedure, which appears in the present paper in line (2.4), where the sublinear functional is $(1+\varepsilon) K T$, and the extended sublinear functional is $d^{+} \phi(z)()$. We have also applied this procedure to obtain a number of other results on the existence of subtangents to proper convex lower semicontinuous functions. These include other generalisations of the maximal monotonicity theorem. (See [6].) There is a feature in this paper that does not appear in [4], [5] or [6]: the existence theorem (Lemma 2) uses $(q, h) \in E \times \mathbb{R}$ such that $\phi(q)>h>\inf \phi(E)$ and the new feature (see Lemma 3 ) is the pushing down of $h$ to the lower end of this range in order to decrease $K(q, h, \phi, T)$. The only place in this paper where we use a result from [4], [5] or [6] is in line (0.3).

DEFINITION of the statement that $\partial \psi$ is locally maximal monotone.

With the notation explained below, this means that if

$$
q \in E, a \in E^{\prime} \backslash \partial \psi(q)
$$

and

$$
U \text { is a convex open subset of } E^{\prime}
$$

such that

$$
U \ni a \text { and } U \cap R(\partial \psi) \neq \emptyset
$$

then

$$
\exists(z, b) \in \partial \psi \text { such that } b \in U \text { and }\langle z-q, b-a\rangle<0 .
$$

What WE Shall PROVE.

In fact, we shall prove the following result.

MaIN Theorem. Let $q \in E, a \in E^{\prime} \backslash \partial \psi(q)$ and $e \in R(\partial \psi)$. Then

$$
[a, e] \cap \overline{\{b: \exists z \in E \text { such that } b \in \partial \psi(z) \text { and }\langle z-q, b-a\rangle<0\}} \neq \emptyset .
$$

The Main Theorem formally implies the following result, which in turn formally implies that $\partial \psi$ is locally maximal monotone. The author is grateful to Professor S. Fitzpatrick for pointing out that statement in the Main Theorem is in fact equivalent to the statement that $\partial \psi$ is locally maximal monotone.

Corollary. Let $q \in E, a \in E^{\prime} \backslash \partial \psi(q)$ and $C$ be a convex subset of $E^{\prime}$ such that $C \ni a$ and $C \cap R(\partial \psi) \neq \emptyset$. Then

$$
C \cap \overline{\{b: \exists z \in E \text { such that } b \in \partial \psi(z) \text { and }\langle z-q, b-a\rangle<0\}} \neq \emptyset .
$$

Other notation. We suppose throughout that $E$ is a real Banach space and $\phi, \psi: E \rightarrow \mathbb{R} \cup\{\infty\}$ are proper, convex and lower semicontinuous.

Let $0<m \leqslant M<\infty$. Let $T: E \rightarrow \mathbb{R}$ be a sublinear functional such that

$$
m\|\| \leqslant T \leqslant M\|\| \text { on } E \text {. }
$$


Write $\tau=m / M . \tau$ measures the "degree of asymmetry" of $T$. We note for future reference that

$$
\tau T \leqslant m\|\| \text { on } E .
$$

Define $T_{q}: E \rightarrow \mathbb{R}$ by

$$
T_{q}(y):=T(q-y) \quad(y \in E) .
$$

$T_{q}$ is a convex, continuous function on $E$. If $q \in E$ and $\phi(q)>h>\inf \phi(E)$, we write

$$
K(q, h, \phi, T):=\sup _{\phi(y)<h} \frac{h-\phi(y)}{T(q-y)}=\sup _{\phi(y)<h} \frac{h-\phi(y)}{T_{q}(y)} .
$$

It was proved in $[4$, Lemma $2.2(\mathrm{a}), \mathrm{p} .130]$ and $[5$, Théorème $2(\mathrm{a})]$ that

$$
0<K(q, h, \phi,\|\|)<\infty \text {. }
$$

If $z \in E$, the subdifferential, $\partial \phi(z)$, of $\phi$ at $z$ is defined by

$$
\left\{c: c \in E^{\prime}, \forall y \in E, \phi(z)-\phi(y) \leqslant(z-y, c)\right\},
$$

where $E^{\prime}$ is the adjoint of $E$. We write $\partial \phi$ for $\{(z, c): z \in E, c \in \partial \phi(z)\} \subseteq E \times E^{\prime}$ and $R(\partial \phi)$ for $\{d: \exists w \in E$ such that $d \in \partial \phi(w)\} \subseteq E^{\prime}$.

If $z \in \operatorname{dom} \phi$ and $v \in E$ then the directional derivative of $\psi$ at $z$ in the direction $v$ is defined by

$$
d^{+} \psi(z)(v):=\lim _{\theta \rightarrow 0+} \frac{\psi(z+\theta v)-\psi(z)}{\theta} .
$$

Since the limit in the above expression can be replaced by an infimum, it follows that

$$
\psi(z+v) \geqslant d^{+} \psi(z)(v)+\psi(z) .
$$

Lemma 1. Let $\alpha, \beta>0, y \in \operatorname{dom} \psi$ and $\psi(y)<\inf \psi(E)+\alpha \beta$. Then

$\exists z \in \operatorname{dom} \psi$ such that $\|z-y\|<\alpha$ and, $\forall v \in E, d^{+} \psi(z)(v) \geqslant-\beta\|-v\|$.

Proof: We choose $\gamma<\alpha$ so that $\psi(y)<\inf \psi(E)+\gamma \beta$. From [1, Theorem 1, p.444] with $\varepsilon:=\gamma \beta$ and $d(u, v):=\|u-v\| / \gamma, \exists z \in \operatorname{dom} \psi$ such that

$$
\|z-y\| / \gamma \leqslant 1 \text { and, } \forall w \in E, \psi(w) \geqslant \psi(z)-\gamma \beta\|z-w\| / \gamma .
$$

The required result follows from the definition of directional derivative by putting $w:=$ $z+\theta v$ and letting $\theta \rightarrow 0+$. 
Lemma 2. Let $q \in E$ and $\phi(q)>h>\inf \phi(E), K:=K(q, h, \phi, T)$ and $\varepsilon \in$ $(0,1)$. Then:

(a) $0<K<\infty$.

(b) $\exists y \in E$ such that

$y \neq q$ and $\left(\phi+K T_{q}\right)(y)<\inf \left(\phi+K T_{q}\right)(E)+\tau \varepsilon m K\|q-y\|$.

(c) $\exists(z, c) \in \partial \phi$ such that

$$
\langle q-z, c\rangle \geqslant(1-\varepsilon) K T_{q}(Z)>0 \text { and } c \leqslant(1+\varepsilon) K T \text { on } E .
$$

Proof: (a) This follows from (0.1) and (0.3).

(b) Let $y \in E$. If $\phi(y) \geqslant h$ then $h \leqslant\left(\phi+K T_{q}\right)(y)$. If $\phi(y)<h$ then

$$
\frac{h-\phi(y)}{T_{q}(y)} \leqslant K
$$

from which, again, $h \leqslant\left(\phi+K T_{q}\right)(y)$. Taking the infimum over $y$,

$$
h \leqslant \inf \left(\phi+K T_{q}\right)(E) .
$$

Since $K\left(1-\varepsilon \tau^{2}\right)<K$, from the definition of $K, \exists y \in E$ such that

from which $y \neq q$ and

$$
\phi(y)<h \text { and } \frac{h-\phi(y)}{T_{q}(y)}>K\left(1-\varepsilon \tau^{2}\right),
$$

that is

$$
\begin{gathered}
K\left(1-\varepsilon \tau^{2}\right) T_{q}(y)+\phi(y)<h, \\
\left(\phi+K T_{q}\right)(y)<h+\varepsilon K \tau^{2} T_{q}(y) .
\end{gathered}
$$

(b) follows by combining this with (0.2) and (2.1).

(c) From (b) and Lemma 1 with $\psi:=\phi+K T_{q}, a:=\|q-y\|$ and $\beta:=\tau \varepsilon m K$, $\exists z \in \operatorname{dom}\left(\phi+K T_{q}\right)=\operatorname{dom} \phi$ such that $\|z-y\|<\|q-y\|$ (thus $z \neq q$, from which $\left.T_{q}(z)>0\right)$ and,

$$
\forall v \in E, d^{+}\left(\phi+K T_{q}\right)(z)(v) \geqslant-\varepsilon K \tau m\|-v\| \geqslant-\varepsilon K \tau T(-v) .
$$

We first prove that,

$$
\forall v \in E, d^{+} \phi(z)(v)+(1+\varepsilon) K T(q-z-v) \geqslant(1-\varepsilon) K T_{q}(z) .
$$

To this end, let $v \in E$. Then since $\tau \leqslant 1$,

$$
d^{+} \phi(z)(v)+(1+\varepsilon) K T(q-z-v) \geqslant d^{+} \phi(z)(v)+K T_{q}(z+v)+\varepsilon K \tau T_{q}(z+v),
$$


from (0.4) (applied to $\psi:=K T_{q}$ ),

$$
\geqslant d^{+} \phi(z)(v)+d^{+} K T_{q}(z)(v)+K T_{q}(z)+\varepsilon K \tau T_{q}(z+v),
$$

from (2.2) (since $\left.d^{+} \phi(z)(v)+d^{+} K T_{q}(z)(v)=d^{+}\left(\phi+K T_{q}\right)(z)(v)\right)$,

$\geqslant-\varepsilon K \tau T(-v)+K T_{q}(z)+\varepsilon K \tau T_{q}(z+v)=-\varepsilon K \tau T(-v)+K T_{q}(z)+\varepsilon K \tau T(q-z-v)$

since $T$ is sublinear,

$$
\geqslant K T_{q}(z)-\varepsilon K \tau T(z-q) \geqslant(1-\varepsilon) K T_{q}(z),
$$

since, from (0.1) and (0.2), $T T(z-q) \leqslant m\|z-q\|=m\|q-z\| \leqslant T(q-z)=T_{q}(z)$. This completes the proof of (2.3). We define a function $S$ on $E$ by

$$
S(w):=\inf \left\{d^{+} \phi(z)(v)+(1+\varepsilon) K T(w-v): v \in E\right\} .
$$

From (2.3), $S(q-z) \geqslant(1-\varepsilon) K T_{q}(z)>0 . S$ is real-valued and sublinear. From the one-dimensional Hahn-Banach theorem, there is a linear functional $c$ on $E$ such that $c \leqslant S$ on $E$ and

$$
\langle q-z, c\rangle=S(q-z) \geqslant(1-\varepsilon) K T_{q}(z)>0 .
$$

Since $c \leqslant S$ on $E, c \leqslant(1+\varepsilon) K T$ and $c \leqslant d^{+} \phi(z)$ on $E$. From (0.1), $c \in E^{\prime}$, hence $c \in \partial \phi(z)$. This completes the proof of (c).

Lemma 3. Let $q \in E$ and $\phi(q)>\inf \phi(E)$. Suppose that $N>0, d \in R(\partial \phi)$ and $d \leqslant N T$ on $E$. Then there is $h$ such that $\phi(q)>h>\inf \phi(E)$ and $K(q, h, \phi, T) \leqslant N$.

Proof: We first suppose that inf $\phi(E)=-\infty$. Let $w \in E$ be such that $d \in \partial \phi(w)$ and write $h:=\phi(w)+\langle q-w, d\rangle-1$. Then $\phi(q)>\phi(q)-1 \geqslant h>-\infty=\inf \phi(E)$. Further, $\forall y \in E$,

$$
\phi(y)+N T_{q}(y) \geqslant \phi(y)+\langle q-y, d\rangle \geqslant \phi(w)+\langle y-w, d\rangle+\langle q-y, d\rangle=h+1 \geqslant h,
$$

from which the result follows. If, on the other hand, $\inf \phi(E)>-\infty$, we first choose $p$ such that $\phi(q)>p>\inf \phi(E)$, then $\delta>0$ so that $(\|q-y\|<\delta \Rightarrow \phi(y)>p)$ and, finally, $h$ so that

$$
\min \{\inf \phi(E)+m \delta N, p\}>h>\inf \phi(E) .
$$

If $\phi(y)<h$ then $\phi(y)<p$, hence $\|q-y\| \geqslant \delta$, from which $T_{q}(y) \geqslant m \delta$. The result follows since $h-\phi(y) \leqslant m \delta N$.

Lemma 4. Let $q \in E$ and $\phi(q)>\inf \phi(E)$. Suppose that $0<N<1, d \in R(\partial \phi)$ and $d \leqslant N T$ on $E$. Then $\exists(z, c) \in \partial \phi$ such that $\langle q-z, c\rangle>0$ and $c \leqslant T$ on $E$.

Proof: We choose $h$ as in Lemma 3. Since $K(q, h, \phi, T) \leqslant N<1$, we can choose $\varepsilon \in(0,1)$ so that $(1+\varepsilon) K(q, h, \phi, T) \leqslant 1$. The result follows from Lemma 2(c). 
LemMa 5. Let $q \in E$ with $\phi(q)>\inf \phi(E), d \in R(\partial \phi)$ and $\eta>0$. Then $\exists(z, c) \in \partial \phi$ and $\lambda \in[0,1]$ such that $\langle q-z, c\rangle>0$ and $\|c-\lambda d\| \leqslant \eta$.

Proof: We define $T: E \rightarrow \mathbb{R}$ by

$$
T(x):=\eta\|x\|+\max \{0,\langle x, d\rangle\}
$$

Then $T$ is sublinear, and $(0.1)$ is satisfied with $m:=\eta$ and $M:=\eta+\|d\|$. We choose $N$ so that

$$
\frac{\|d\|}{\eta+\|d\|}<N<1
$$

Then $d \leqslant N T$ on $E$. We find $(z, c) \in \partial \phi$ as in Lemma 4. Let $A$ be the weak* compact convex subset $\left\{e+\lambda d: e \in E^{\prime},\|e\| \leqslant \eta, \lambda \in[0,1]\right\}$. Then,

$$
\forall x \in E, T(x)=\max \langle x, A\rangle
$$

Since $c \leqslant T$ on $E$, from the bipolar theorem, $c \in A$. This gives the required result. $\square$

Lemma 6. Let $q \in E$ with $\phi(z)>\inf \phi(E)$ and $d \in R(\partial \phi)$. Then

$$
[0, d] \cap \overline{\{c: \exists z \in E \text { such that } c \in \partial \phi(z) \text { and }\langle q-z, c\rangle>0\}} \neq \emptyset \text {. }
$$

$([0, d]$ stands for the line segment joining 0 and $d$.

ProOf: From Lemma 5, $\forall n \geqslant 1, \exists\left(z_{n}, c_{n}\right) \in \partial \phi$ and $\lambda_{n} \in[0,1]$ such that $\left\langle q-z_{n}, c_{n}\right\rangle>0$ and $\left\|c_{n}-\lambda_{n} d\right\| \leqslant 1 / n$. Let $\lambda$ be a limit point of $\left\{\lambda_{n}\right\}_{n} \geqslant 1$. Then $\lambda d$ is in the intersection in the statement of the lemma.

Ma In Theorem. Let $q \in E, a \in E^{\prime} \backslash \partial \psi(q)$ and $e \in R(\partial \psi)$. Then

$$
[a, e] \cap \overline{\{b: \exists z \in E \text { such that } b \in \partial \psi(z) \text { and }\langle z-q, b-a\rangle<0\}} \neq 0 \text {. }
$$

Proof: Let $\phi:=\psi-a$. Then $0 \notin \partial \phi(q)$, hence $\phi(q)>\inf \phi(E)$. Let $d:=e-a$. Then $d \in R(\partial \phi)$. If $f$ is in the intersection of Lemma 6 then $a+f$ is in the intersection in the statement of the Theorem.

\section{REFERENCES}

[1] I. Ekeland, 'Nonconvex minimization problems', Bull. Amer. Math. Soc. 1 (1979), 443-474.

[2] S. Fitzpatrick and R.R. Phelps, 'Bounded approximants to monotone operators on Banach spaces', Ann. Inst. H. Poincaré Anal. Non Linéaire $\theta$ (1992), 573-595.

[3] J.-P. Gossez, 'On a convexity property of the range of a maximal monotone operator', Proc. Amer. Math. Soc. 55 (1976), 359-360. 
[4] S. Simons, "The least slope of a convex function and the maximal monotonicity of its subdifferential', J. Optim. Theory Appl. 71 (1991), 127-136.

[5] S. Simons, 'Les dérivées directionnelles et la monotonicité maximale des sous-différentiels', Séminaire d'Initiation à l'Analyse (Séminaire Choquet) (to appear).

[6] S. Simons, 'Subtangents with controlled slope'. (Submitted).

Department of Mathematics

University of California

Santa Barbara CA 93106-3080

United States of America 